\title{
ERRATUM
}

\section{Lineage dependency and lineage-survival oncogenes in human cancer}

Levi A. Garraway and William R. Sellers

Nature Rev. Cancer 6, 593-602 (2006)

On page 596 of this article the statement 'The lineage-dependency model (FIG. 1) therefore offers a counterpoint to oncogene addiction...' should have cited Figure 3 rather than Figure 1. The online version of the article has been corrected. 